\title{
A novel HSP90 inhibitor, 17-DMAG, induces Tax down-regulation and its oral administration to ATL-model mice intervenes against the infiltration property of the ATL-like lymphocytes and provides extended survival period
}

\author{
Emi Ikebe ${ }^{1}$, Akira Kawaguchi ${ }^{2,6}$, Kenta Tezuka ${ }^{3}$, Shinya Taguchi', Satoshi Hirose ${ }^{1}$, Takashi Matsumoto ${ }^{1}$, \\ Takahiro Mitsui ${ }^{1}$, Kazuyo Senba', Akira Nishizono ${ }^{1}$, Mitsuo Hori ${ }^{4}$, Hiroo Hasegawa ${ }^{5}$, Yasuaki Yamada ${ }^{5}$, \\ Takaharu Ueno ${ }^{3}$, Yuetsu Tanaka ${ }^{6}$, Hirofumi Sawa ${ }^{7}$, William Hall ${ }^{8}$, Yasuaki Minami ${ }^{9}$, Kuan-Teh Jeang ${ }^{10}$, \\ Masao Ogata ${ }^{11}$, Kazuhiro Morishita ${ }^{12}$, Hideki Hasegawa ${ }^{2}$, Jun-ichi Fujisawa ${ }^{3}$, Hidekatsu Iha ${ }^{*}$
}

From 16th International Conference on Human Retroviruses: HTLV and Related Viruses Montreal, Canada. 26-30 June 2013

In the peripheral blood leukocytes (PBL) infected with human T-cell leukemia virus type-1 (HTLV-1), which causes HTLV-1 associated diseases including adult T-cell leukemia (ATL), HTLV-1 associated myelopathy (HAM) and HTLV-1 uveitis (HU), NF- $\kappa$ B-mediated anti-apoptotic signals or inflammatory signals are constitutively activated primarily by the HTLV-1 encoded oncoprotein Tax.

Tax interacts with the I- $\kappa \mathrm{B}$ kinase regulatory subunit, $\mathrm{NEMO}$, to activate NF- $\kappa \mathrm{B}$, and this interaction is maintained in part by a molecular chaperone, Hsp90, and its co-chaperone Cdc37. The antibiotic geldanamycin (GA) inhibits Hsp90's ATP binding for its proper interaction with client proteins. Administration of a novel water soluble and less toxic GA derivative, 17-dimethylaminoethylamino-17demethoxygeldanamycin hydrochloride (17-DMAG) to Tax-expressing ATL transformed cell lines, C8166 and MT4, induced significant degradation of Tax. 17-DMAG also facilitated growth arrest and cellular apoptosis to C8166 and MT4 and other ATL cell lines while this treatment has no apparent effects on normal PBLs. 17-DMAG also down-regulated Tax-mediated intracellular signals including activation of NF- $\kappa \mathrm{B}, \mathrm{AP}-1$ or HTLV1-LTR in Tax-transfected HEK293 cells.

'Department of Infectious Diseases, Oita University, Faculty of Medicine, Japan Full list of author information is available at the end of the article
Oral administration of 17-DMAG to ATL-model mice xenografted with lymphomatous transgenic Lck-Tax cells or HTLV-1 producing tumor cells dramatically attenuated the aggressive infiltration into multiple organs, viral replication and improved survival periods. These observations identified 17-DMAG as a promising candidate for prevention of ATL progression.

\section{Authors' details}

'Department of Infectious Diseases, Oita University, Faculty of Medicine, Japan. ${ }^{2}$ Department of Pathology, National Institute of Infectious Diseases, Japan. ${ }^{3}$ Department of Microbiology, Kansai Medical University, Japan. ${ }^{4}$ Department of Hematology, Ibaragi Pref. Central Hospital, Japan. ${ }^{5}$ Department of Laboratory Medicine, Nagasaki University Graduate School of Biomedical Sciences, Japan. ${ }^{6}$ Department of Immunology., Graduate School of Medicine University of Ryukyus, Japan. ${ }^{7}$ Department of Molecular Pathobiology, Hokkaido University Research Center Zoonosis Control, Japan. ${ }^{8}$ Department of Medicine and Microbiology, Centre Research Infectious Disease, Conway Institute Biomolecular Biomedical Research University, Coll. Dublin, Ireland. ${ }^{9}$ Department of Biotechnology, Maebashi Institute Technology, Japan. ${ }^{10}$ Laboratory and Molecular Microbiology, National Institutes of Health, USA. " Department of Hematology, Oita University, Faculty of Medicine, Japan. ${ }^{12}$ Department of Medical Sciences, Faculty of Medicine, University of Miyazaki, Japan.

Published: 7 January 2014

\section{doi:10.1186/1742-4690-11-S1-P44}

Cite this article as: Ikebe et al: A novel HSP90 inhibitor, 17-DMAG, induces Tax down-regulation and its oral administration to ATL-model mice intervenes against the infiltration property of the ATL-like lymphocytes and provides extended survival period. Retrovirology 2014 11(Suppl 1):P44. 